\title{
Underground Cable Fault Detection Using Internet of Things
}

\author{
Sathana.B ${ }^{\# 1}$, Jaissandiya.R ${ }^{\# 2}$, Divyadarshni.S $S^{\# 3}$, Ast.Prof. Poonguzhali.E.$^{\# 4}$ \\ UG - Information Technology, Sri Manakula Vinayagar Engineering College, Pondicherry, India ${ }^{1-3}$ \\ Associate Professor, Information Technology, Sri Manakula Vinayagar Engineering College, Pondicherry, India ${ }^{4}$
}

\begin{abstract}
Internet of Things is an Internet-connected object system that can store and transmit data on a wireless network without human interference. Internet of Things is a wireless system. IoT has its major contribution in fault diagnosis and prediction of the physical devices by analyzing the device without the knowledge of the physical manufacturing system Underground cables, due to underground stresses, wear and tear, rodents, etc, are subject to a variety of defects. It is also difficult to detect fault sources. To inspect and repair the failure, the whole line has to be dug. We, therefore, propose an Underground Cable Fault Detector using IoT that detects the exact position of the defect and simplifies the repair. To locate the root of the problem, the repairmen know which component is defective and only the region must be dug. This saves a lot of time, money, and effort and enables simple underground cable maintenance. This saves a great deal of time, money, and effort and allows for easy cable maintenance in the underground. We use Ohm's law principle and Blavier's test to detect and verify failures over the internet by authorities, here the Arduino board that is an IoT component functions as a machine brain and handles the sensor data. The machine detects errors by using the future cable-wide divisor network. When a failure occurs when two lines are cut, a certain voltage will be generated according to a combination of the resistance network. The microcontroller senses this voltage and is modified. The information the consumer receives is the distance that corresponds to this voltage. The microcontroller collects fault line data and displays it over an LCD monitor so that this data is transferred to the internet for online access.
\end{abstract}

Keywords - Underground cables, Ohm's Law, Arduino, Blavier's test.

\section{INTRODUCTION}

Long transmission lines and overhead lines were an "indissoluble binomial" for AC Power Systems in the early years. Faults are described as the complete failure or loss of synchronization of a power system network, which does not preclude environmental risks such as electrocution and a catastrophic fire outbreak. This indicates that the general belief at the time was that signal transmission was primarily based on overhead lines. High Voltage (HV) and Extra High Voltage (EHV) insulated cables were left to be used for DC submarine connections. This was due, among other things, to a greater awareness of environmental conditions, growing obstacles encountered on overhead lines, and increased reliance on high-quality extruded insulations. Power systems operators in the power sectors in different countries have considered replacing these overhead cables and lines with underground ones or implementing a hybrid system (i.e. combining overhead lines and underground cables).The majority of underground cable system installations are carried out for a variety of reasons, including cost. The following are some of the benefits of having it mounted.

1. A significantly decreased risk of damage from environmental hazards such as lightning, winds, and freezing, to name a few.

2. An underground cable system reduces the spectrum of Electromagnetic Fields (EMF) that can be emitted.

3. Alongside the underground cables, fewer components are installed. In the case of overhead lines, this is the reverse, when more components are placed alongside them for protection, maintenance, or repair.

4. The underground cable system removes the risk of danger to flying aircraft and wildlife.

5.Stealing, sabotage, and unauthorized contacts of conductors are less like 6. In environmentally conscious countries, an underground cable system may be used to plant and grow large trees.

In certain cases, the benefits of the underground system phase can outweigh the disadvantages. If a failure occurs, one of the most obvious and realistic drawbacks of the underground system method is the difficulty in finding it. Underground cables have been used in several decades by much of the world's low and medium voltage distribution lines. Underground cables with high voltage are used more and more because the weather, heavy rain, storm, snow, and pollution do not affect them. Though cable production technology is continuously improving, factors that can lead to the failure of the cable during testing and operation still exist. A decent cable will last for around 30 years in good condition and properly mounted. Cables can however be quickly destroyed by incorrect installation or malfunctioning whereas civil works such as trenching or curbing can damage subsequent third parties.

\subsection{Internet of Things}




\section{International Advanced Research Journal in Science, Engineering and Technology}

Vol. 8, Issue 7, July 2021

\section{DOI: 10.17148/IARJSET.2021.8709}

The Internet of Things (IoT) is a network of interconnected, internet-connected devices that can capture and transmit data without the need for human interaction over a wireless network. The Wi-Fi module is used in IoT to view information over the Internet. The details about the occurrence of the fault are displayed on a web page created with HTML coding. The short circuit fault is situated at a fixed distance in the underground cable to effectively rectify the fault using basic Ohms law principles. The repairmen know exactly which component is faulty, and only that area needs to be dug up to locate the root of the problem. As a result, we save a lot of time and money, and we can service underground cable lines more quickly. We use the Internet of Things (IoT) technology, which helps authorities track and verify faults over the internet. Fault Detector for Underground Cables Using IoT. Owing to underground temperatures, wear and tear, rats, and other factors, underground cables are susceptible to a wide range of faults. Diagnosing the cause of a fault is challenging because to inspect and repair faults, the whole cable must be removed from the ground. The manual process takes a long time. We propose a cable fault detection over IoT that detects the exact fault location over IoT and simplifies the repair process.

\subsection{Power Supply}

The power supply circuit includes a step-down transformer that converts 230 volts to 12 volts. In this circuit, four diodes are used to create a bridge rectifier that delivers pulsating dc voltage, which is then fed to a capacitor filter, which eliminates any a.c. components present even after rectification. The filtered DC voltage is fed into the regulator, which produces a steady DC voltage of 12 volts.

\subsection{Rectifier}

The rectifier receives the transformer's production. It transforms alternating current into pulsating direct current. The rectifier may be either half-wave or full-wave. Because of its advantages, such as good stability, a bridge rectifier is used in this project. The circuit is made up of four diodes that form a bridge. A rectifier is an electrical device that transforms alternating current (AC), which reverses direction regularly, to direct current (DC), which only flows in one direction. The procedure is referred to as rectification. Rectifiers have a wide variety of applications, but they're most commonly used as parts of DC supplies and high-voltage direct-current power transmission systems. Rectification may be used for purposes other than producing direct current for use as a power source. 5.3-inch LCD The microcontroller 8051 is connected to a liquid crystal display. The most popular LCDs are $16 * 2$ and $20 * 2$. In a $16 * 2$ display, 16 denotes a column, and 2 denotes a row. LCDs can show random images (as in a general-purpose computer display) or fixed images with low information content that can be seen or obscured, such as preset words, digits, and 7-segment displays in a digital clock. They share the same basic technologies, with the exception that random images are made up of a large number of tiny pixels, while other displays have larger components.

\subsection{Voltage Regulator}

A voltage controller is an electrical controller designed to keep the voltage steady. The power supply for this project is $5 \mathrm{~V}$ and $12 \mathrm{~V}$. To obtain these voltage levels, 7805 and 7812 voltage regulators are to be used. The first number 78 is positive, and the numbers 05,12 reflect the voltage levels needed for the output. Three terminal positive regulators are available in the L78xx series.

\subsection{Arduino}

LCDs Arduino is an open-source forum for construction projects in electronics. Arduino consists of both a physical programmable circuit board (often referred to as a microcontroller) and a piece of software, or IDE (Integrated Development Environment) that runs on your computer, used to write and upload computer code to the physical board. The Arduino platform has become quite popular with people just starting with electronics, and for good reason. Unlike most other programmable circuit boards, Arduino does not require separate hardware (called a programmer) to load the new code onto the board. Additionally, the Arduino IDE uses a simplified version of $\mathrm{C}++$, making it easier to learn to program. Arduino finally offers a standard form factor that splits the microcontroller's functions into a more usable kit.

\subsection{Relay}

Relay is a sensing device that senses the fault and sends a trip signal to the circuit breaker to isolate the faulty section. A relay is an automated system that indirectly controls the electrical circuit and controls changes in the same or another electrical circuit. There are different kinds of relays: numerical, static, and electromagnetic relays. Relays are housed in panels in the control room. Each of the three phases uses three mini power relays. The relays scan the three phases regularly and forward the signal to the Arduino controller. Each relay has a rating of approximately $12 \mathrm{~V}$.

\subsection{Relay Driver IC}

The Driver Circuit boosts or amplifies signals from microcontrollers to monitor power switches in semiconductor devices. Driver circuits perform tasks such as isolating the control and power circuits, detecting malfunctions, storing and reporting faults to the control system, acting as a fail-safe, analyzing sensor signals, and generating auxiliary voltages. The relay driver circuit in this project is the ULN2003. It's an unconnected circuit that acts as a relay driver and boosts the relay's power supply.

\section{REFERENCE}




\section{International Advanced Research Journal in Science, Engineering and Technology}

Vol. 8, Issue 7, July 2021

\section{DOI: 10.17148/IARJSET.2021.8709}

Various approaches have been proposed to detect the faults in underground cables, but later proved flawed. This paper [1] presents experimental observations of the characteristics of surge propagation on different cables, i.e. overhead, on the floor, on the ground floor, underground and underwater. The calculated results show clearly that overhead or underground coaxial mode of surge propagation is not known to rely on either the outer cable medium. An earth return mode depends heavily on the external medium. EMTP experimental simulations have been carried out and, compared to experimental studies, the simulation results indicate adequate accuracy. Cables consisting of underwater and submarine / overhead cables are investigated based on EMTP simulation data, considering cable cross-bonding. Surge characteristics of a cable system The cross-connection leads to a higher surge voltage. The sheath overvoltage in a cross-bound cable in particular is far higher. Cross-bonding in a submarine component, therefore, should not be used that costs a lot for reinstallation. This paper is intended to provide proper reasoning as to why the cables are buried underground. The study suggests in the idea [2] an essay on the origin and use of underground cables. Underground power cables constitute a necessary connection between two or more electrical devices. A great number of materials, designs, and constructions have been developed due to the various applications, service requirements, and technological methods related to the development and installation of insulated power cables. The power cables for the power distribution, transmission, and distribution of electricity consist primarily of conductors stranded by metals of high conductance such as copper or aluminium isolated from a central conductor using fluid-impregnated force-paper tapes or polymer-based materials. Underground cables are suitable for areas in which some obstacles and hazards make it impossible to use overhead lines. In comparison with the overhead cables, the drawback is that the underground cable lines are much higher or moderate in price. The work presented in [3] proposed underground Arduino cable defect detection. The project seeks a package of the Arduino microcontroller to detect errors in underground cable lines in the kilometer from the base station to the exact site. A series of switches makes the formation of the fault. Control of relays through the relay motor. The $16 x 2$ LCD monitor for displaying information is connected to the microcontroller. If the voltage changes over the series resistor when a short circuit is fed to an ADC to develop exact digital information into the Arduino programmed microcontroller kit, which shows the precise position of the fault from the base station for kilometres further. Whenever a failure takes place in a wire, the buzzer alerts field staff and acts immediately. The Arduino microcontroller functions based on cable strength performance. Relay helps to distinguish the faulty line from the good line. This project only identifies where the circuit error is located in the underground cable. The Author suggested a method for finding the exact position of the fault in the work implemented in [4]. The prototype is modeled on a set of resistors that reflect a kilometer-long cable, and failure detection is carried out by a set of switches to verify its accuracy at any known distance. The short circuit fault in the underground cable at a certain distance is located to correct the fault effectively with the basic principles of Ohm's rule. The work is not finished for open circuit failure, line by line failure, and ground fault line by line. The author has introduced a method in which the position of the defect in underground cable lines is detected, to concentrate on [5]. The method proposed finds the exact position of the open-circuit defect. The benefit of this project is that cable life can be enhanced because the power supply transmission can be stopped before the failure is fixed, thus avoiding excessive losses due to current leakage. Unable to know the form of fault. The concept put in [6] suggested the underground detection of cable defects using IoT, where underground cables are susceptible to various defects due to the underground, wear and tear, rodents, etc. It is difficult to diagnose the fault source and remove the entire cable from the ground to inspect and remedy failures. This project aims to detect fault locations from the base station in underground cable lines in kilometres using a PIC16F877A controller. A 16X2 LCD interfacing with the microcontroller shows the size, phase, and time faults. IoT uses the Wi-Fi module ESP8266 to view information on the Internet. An HTML coding is used to build a webpage and information on failure is available on a webpage. Quick repair to restore the power supply, increase system efficiency and reduce the running expenses and time for locating the errors in the field is the advantage of the precise position of the fault. In the proposed work in [7], the Author proposed an automated subterranean IOT cord failure detector to design the numerical relay, when the input value exceeds the reference value in the relay that then transfers the travel signal to the circuit interrupter. It finds the position or location of the fault and also the exact distance from the breaker. The detector can only calculate a certain distance in the event of a defect. The author proposes the design and implementation of an underground cable fail-detector in the form of a model built in [8], where the detector can measure cable resistance, detect fault type in a cable and accurately calculate fault position with inexpensive materials. The device has the capability of measuring the strength of the cable, the type of failure in the cable and the position of the fault with cheap materials can also be precisely calculated. Concerning open and short circuit problems in underground cables with a maximum distance of $2 \mathrm{~km}$, the proposed procedure obtained successful results. In the future, the effort to raise the maximum distance to detect failures to $3 \mathrm{~km}$ or more is focused and the LCD may be replaced by a graphical display monitor to enhance its knowledge on the underground cable fault. In the model implemented in the study [9], the purpose of this project is to evaluate the subterranean cable fault distance in $\mathrm{km}$ from the base station. The main objective of this research project is to detect underground cable faults. Cable defects are cable damage that affects the cable resistance. This will result in a voltage breakdown if it will last. The cable must first be checked for errors to detect a cable error. This project uses the basic notion of the law of ohm. If a defect such as the short circuit happens, the voltage drop is different according to the length of the cable fault, since the current is different. Therefore, a series of resistors is used to reflect a cable and a dc voltage is fed at one end of the system and the fault is detected by using the analog voltage 


\section{International Advanced Research Journal in Science, Engineering and Technology \\ Vol. 8, Issue 7, July 2021 \\ DOI: 10.17148/IARJSET.2021.8709}

converter to detect the voltage variations. To calculate the fault distance on the LCD monitor, a microcontroller is employed. The detector must be sufficiently similar to detect defects best. The author developed the IoT-based Fault Detection diagnosis in the GOOGLE Firebase transmission in [10]. It is undoubtedly hazardous for the territory when the event occurred at the transmission line. There is less problem in the HV and EHV lines but, if compared with external transmission lines, the deficiency event in the area is more significant. We structure in our model a model that is supposed to distinguish the lack of the transmission line by looking at the transformer's MCU node, if power passes through this, when the following signal goes to GOOGLE Firebase database, the transformer's number and corresponding MCU node is determined. The data for the particular event is sent to the page through the IoT gadget which is NODE MCU(Esp8266) and which has additionally been displayed. This IC writing computer programmers uses the NODE microcontroller, which also reflects on the voltage signal, sends out the output, and displays it to the IoT module. The power supply is supplied to all segments of this stock with a capacity of 3,3-volt dc, separated from the stock used to monitor the issue.

\section{CONCLUSION}

The problem of fault position in an underground cable power grid has received a lot of attention. However, a simple ohm's law-based technology has been developed to quickly locate faults in an underground power cable layout, which inevitably aids in 8yfault clearing, maintaining aesthetics, reducing time and drudgery, and maximising cost. The advances in underground cable detector design could lead to widespread adoption of underground cable technologies in developing countries' major cities, reducing environmental disasters associated with overhead transmission lines.

\section{REFERENCES}

1. Yo Goto,Akihiro Ametani,Toshihiro Kubo,Naoto Nagaoka,Yoshihiro Baba 'A surge analysis of a cable system composed of submarine and underground / overhead cables', IEEE,(2009).

2. Professor John Tanaka 'History of Underground Power Cables',IEEE(2013)

3. Raghu Raja Kalia, Preeti Abrol, 'Design and implementation of wireless live wire fault detector and protection in remote areas', IEEE,(2014),vol. 97,No. 17

4. PreetiJaidka, Shreeya Srivastava, JSS Academy of Technical Education, Noida, "Underground Cable Fault Detector Using Arduino" IJEECS ISSN 2348-117X Volume 5, Issue 5 May (2016).

5. T.Nandhini, J.Shalini,T. Sai Sangeeetha,D.Gnanaprakasam 'Underground Cable Fault Detection using Arduino',International Journal of Engineering Science and Computing(IJESC),(2017).

6. Abhay Sharma, AkashMathur, Rajat Gupta, R.B.S. Engineering Technical Campus, Bichpuri, Agra, India, "Underground Cable Fault Distance Locator", IJAREEIE,Vol. 6, Issue 4, April (2017).

7. Faizan Mohammad Yakub, Dinesh Rojatkar, Govt.College of Engg. Chandrapur, Maharashtra, India,"Arduino Cable Fault Detector “A Survey”, January2017, Volume 4, Issue 01 JETIR

8. AkashJagtap, JayeshPatil, BhushanPatil, GF's G.C.O.E Jalgaon, Maharashtra, India, “Arduino based Underground Cable Fault Detection”, ISSN : 2454-9150 Vol-03, Issue 04, May (2017).

9. T.Nandhini, J.Shalini, T. Sai Sangeetha, Dr. Mahalingam College of Engineering and Technology, NPT - MCET Campus, Tamil Nadu, India, "Underground Cable Fault Detection using Arduino", IJESC, Volume 7 Issue No.4,(2017).

10. Mr. N. Sampathraja, Ms. V. Kirubalakshmi and Ms. C. Muthumaniyarasi, Dr. L. Ashok Kumar, 'Iot Based Underground Cable Fault Detector' INTERNATIONAL JOURNAL OF MECHANICAL ENGINEERING AND TECHNOLOGY ,(IJMET),(2017).

11. Dr. H. Umadevi,Niketh B.Vijaydeep,Vikas TV, 'Underground Cable Fault Monitoring \& Detection System using IoT \& Arduino' ,Journal for research,(2018).

12. Henna Sam.E.J, Pavithra.U.H, Ramakrishnan.P.V, Riyash Basha.S and Mr.T.M.Sathish kumar, 'Underground Cable Fault Detection Using IoT', Asian Journal of Applied Science and Technology(AJAST), (2018).

13. Ankit S. Gaulkar, Karunesh V. Gaurkar, Kajal V. Dhoble, Prachita K. Yerane, Prof. Nisha Warambhe, 'Result Paper of Automatic Underground Cable Fault Detector Using IOT', MANTECH Publications (2019).

14. Emmanuel Gbenga Dada, Abdulkadir Hamidu Alkali, Stephen Bassi Joseph, Umar Abba Sanda, 'Design and Implementation of Underground Cable Fault Detector', International Journal of Science and Engineering Investigations (IJSEI), (2019).

15. Harshit Srivastava; Naveen Mishra; Prajjwal Srivastava, 'Underground Cable Fault Detection',International Journal of Computing and Technology(IJCAT),(2019).

16. Lakshmi Goswami and Piyush Agrawal, 'IOT based Diagnosing of Fault Detection in Power Line Transmission through GOOGLE Firebase database', Institute of Electrical and Electronics Engineers (IEEE) (2020).

17. Prakash T; Arun G; Arun Kumar B; Deepan S; Kalyana Venkata Ramanan. "IoT Based Smart Irrigation for Multi Cropping System". International Research Journal on Advanced Science Hub, 2, 4, 2020, 7-12. doi: 10.47392/irjash.2020.20

18. Neelam Yadav; Sunil K. Singh. "Implementation of Smart Helmet for Bikers". International Research Journal on Advanced Science Hub, 3 , Special Issue ICARD-2021 3S, 2021, 135-139. doi: 10.47392/irjash.2021.081

19. Soundharya B M; Yogeshwaran R; Tharanish Krishna A D; Vinitha V. "Automatic Check Post and E-Toll Payment System". International Research Journal on Advanced Science Hub, 3, Special Issue ICARD-2021 3S, 2021, 57-62. doi: 10.47392/irjash.2021.064

20. Jijusasikumar S; Kaviya K; Logida R; Chinmaya S; Sangeetha K. "Gas Leakage Monitoring System Using IOT". International Research Journal on Advanced Science Hub, 3, Special Issue ICARD-2021 3S, 2021, 108-111. doi: 10.47392/irjash.2021.075

21. Deshmukh K P. "Wireless Transceiver Module HC-12 based Automatic Water-level Monitoring and Control System". International Research Journal on Advanced Science Hub, 2, 10, 2020, 24-28. doi: 10.47392/irjash.2020.184 